\title{
El sector publicitario a examen: comparativa Valladolid-Castilla y León-España
}

\section{The Advertising Sector to review: comparison between "Valladolid- Castille and Leon" and Spain}

\author{
Begoña Gómez Nieto. Universidad de Valladolid \\ Cristina Benito Vielba. Universidad Europea Miguel de Cervantes
}

Recibido: 15-II-2013 - Aceptado: 21-I-2014

Resumen:

El siguiente artículo se centra en el análisis del sector publicitario (compuesto en su mayoría por pymes) en Castilla y León y concretamente en Valladolid. Se define el sector de la comunicación y la publicidad, la tipología de empresas que ofrecen estos servicios y, en última instancia, la actitud y el comportamiento de las pymes hacia los mismos. Como metodología, se desarrolla una investigación de tipo cuantitativo, concretamente se utiliza la encuesta con cuestionario autoadministrado. Entre los resultados más relevantes destacan: el sector alimentación se consolida como el sector que más invierte en publicidad, los profesionales del sector publicitario analizado poseen formación específica e Internet se conforma como el medio más solicitado por las pymes.

Palabras clave:

Agencia de publicidad; Valladolid; Castilla-León; pyme; inversión publicitaria.

Abstract:

The following article focuses on the analysis of the advertising industry (mostly composed of SMEs) in Castille and Leon and, specifically, in Valladolid. Communication and Advertising industry are defined, as well as the typology of companies which offer these services and, finally, the attitude and behaviour of SMEs towards those companies. As methodology, a quantitative research is developed, specifically, it is used the self-administered questionnaire survey. Some of the most relevant foundings are: food industry is the sector with the largest investment in advertising, the professionals who work in the analyzed advertising sector own specific studies and, finally, Internet is the most requested media by SMEs.

Keywords:

Advertising agency; Valladolid; Castille and Leon; SMEs; advertising investment. 


\section{Introducción}

El sector publicitario en nuestro país se conforma como un motor importante del desarrollo económico. Según el informe Infoadex del año 2013, la inversión real estimada que registró el mercado publicitario se situó en un volumen de 10.858,8 millones de euros, teniendo en cuenta que esto supuso respecto al año anterior un decrecimiento del -9,9\%.

El Ministerio de Educación de España (2012) define la agencia publicitaria como "una empresa especializada en publicidad. Estas empresas sirven a los anunciantes, sus clientes, que pueden ser desde cualquier tipo de empresa, asociación, institución o Administración pública hasta cualquier particular que decida contratar sus servicios para asegurar los resultados de las acciones publicitarias que realice”. Tal y como señala el Artículo 10 de la Ley General de Publicidad 34/1988 de 11 de noviembre, "son agencias de publicidad las personas naturales o jurídicas que se dediquen profesionalmente y de manera organizada a crear, preparar, programar o ejecutar publicidad por cuenta de un anunciante".

Una de las peculiaridades de las agencias de publicidad con respecto a los negocios que ofrecen productos tangibles, es que los profesionales crean cada una de las acciones o productos según los requerimientos y peticiones de cada cliente, por lo que es poco frecuente que ya dispongan de soluciones preparadas con antelación. Cada cliente es único, así como sus características, actividad, necesidades, objetivos o preferencias, por lo que el servicio del que le proveerán también lo será.

Las agencias de publicidad pueden clasificarse según distintos criterios. El Ministerio de Educación, señala como características diferenciadoras su tamaño, el ámbito geográfico en el que desarrollan su actividad, su filosofía de trabajo y el servicio que prestan. En concreto, especifica distintos tipos según este último criterio, el servicio prestado, y señala dos tipos: las agencias de servicio completo o servicios plenos y las agencias especializadas.

Otra clasificación distingue entre las "decanas de la publicidad", refiriéndose a aquellas agencias que nacieron en la década de los 80 e inicios de los 90 y que han contribuido "al crecimiento de la publicidad", a la "dignificación de las ciudades" y al propio "desarrollo social”. Son escasas en Castilla y León; algunos ejemplos en Valladolid serían RQR Comunicación o SM2 (Fernández Blanco, 2011:31).

La siguiente tipología es la de "agencias consolidadas en el mercado", las cuales llevan más de una década de trayectoria, ya que su creación se remonta a finales de los 90 y primeros años 2000. Es en los años 90 cuando Castilla y León asiste a un boom publicitario, con la apertura de un gran número de agencias.

Por último, nos encontramos con las agencias creadas a partir de 2005 o "agencias de reciente creación". En estas agencias se aprecia aún más esa especialización en áreas concretas, como el diseño o la producción web e, incluso, en sectores concretos de actividad, como agroalimentario o entidades sin ánimo de lucro. 
En general son las más afectadas por la crisis, debido a su menor trayectoria y experiencia.

En este artículo profundizamos en los tipos de agencias de publicidad existentes en nuestro entorno y en cómo es la actividad de trabajo de dichos profesionales de la comunicación, teniendo en cuenta que en la mayoría de casos funciona el binomio pyme-agencia, y como muestra la investigación un porcentaje alto de las empresas de publicidad analizadas son pymes.

\subsection{Estado de la cuestión}

\subsubsection{El sector publicitario}

En los últimos años, las agencias de servicios plenos se han visto en la necesidad de mejorar su eficacia y afianzar el lugar que les corresponde en el mercado. Una de las tendencias más predominantes ha sido la progresiva cesión de parcelas de actividad. Esta cesión ha afectado, sobre todo, a las áreas de planificación de medios y producción. Ambas son costosas (tanto a nivel de tiempo como a los costes económicos) y pueden subcontratarse a empresas especializadas (agencias de medios y productoras). De hecho, con el objetivo de mejorar sus servicios y, a su vez, asegurar la supervivencia de su negocio, algunas agencias han creado o han pasado a formar parte de los denominados "Grupos de Comunicación". Éstos están integrados por la propia agencia publicitaria y otras empresas que proveen de prácticamente todos los servicios de marketing y comunicación que pueda precisar cualquier anunciante. Hoy en día no nos extrañan los magníficos resultados que puede obtener un vídeo «casero» colgado en Youtube. Lo mismo puede ocurrir con una estrategia de marketing viral y tantas otras acciones que demuestran que hoy más que nunca el valor de las ideas está muy por encima de la inversión en medios que se realice.

Una asociación de referencia en el mercado publicitario español es la Asociación Española de Agencias de Comunicación publicitaria, fundada en 1977. La AEACP cuenta, en este momento, con 39 miembros, entre los cuales encontramos agencias de prestigio y renombre nacional e internacional como McCann Erickson, Sra. Rushmore, Young \& Rubicam o Saatchi \& Saatchi. Esta Asociación produce, sin una periodicidad precisa, distintos estudios y publicaciones relacionadas con el mundo de la publicidad y la comunicación y señala en su página web que las agencias de publicidad socias de la AEACP facturan en torno al $85 \%$ de la inversión gestionada a través de agencias en España, lo que indica el alto nivel de representación de la asociación en el sector de las agencias.

Según la Asociación General de Empresas de Publicidad (AGEP, 2010), el número de empleados medio de la muestra de empresas ha resultado elevado, con una media de 16,82 trabajadores por empresa, si bien este dato presenta una desviación típica de 34,89 debido a la dispersión de la muestra. 
Los principales servicios ofrecidos son por este orden: publicidad, artes gráficas, carteles, vallas y marquesinas, prensa, marketing, eventos, ferias y mailings. En cuanto al ámbito geográfico en el que se prestan los servicios, las empresas de marketing promocional son las que presentan en mayor porcentaje presencia en el ámbito internacional con un $22,2 \%$ de menciones. Le sigue en este sentido las empresas de publicidad exterior con un 20,8\%. Respecto a los sectores con los que se trabaja fundamentalmente, el sector de las tecnologías de la información ocupa la primera posición (65,8\%), seguido de la banca (17,6\%), alimentación (15,5\%), automoción (13,6\%), seguros (11,5\%) y limpieza e higiene $(6,1 \%)$.

El reparto nacional de empresas de publicidad favorece claramente a la Comunidad de Madrid, que conjuntamente con Cataluña, Andalucía y Comunidad Valenciana explican casi el 75\% del tejido empresarial español. Del total de 8.100 empresas recontadas en nuestra estadística, Galicia cuenta con 293, explicando un 3,6\% del mercado español. Su posición en el ranking por número de empresas es preeminente, sólo superada por País Vasco, Comunidad Valencia, Andalucía, Cataluña y Madrid (Informe Sector de la publicidad, 2007).

La publicidad on line crecerá en España un 4,9\% en el periodo 2013-2017 hasta alcanzar los 1.510 millones de dólares, lo que la sitúa como la segunda industria con mayor crecimiento del mercado de los medios. Sin embargo, la penetración de la banda ancha en España (72\%) es algo más baja que en el resto de Europa, lo que inevitablemente supone una barrera para su crecimiento. Así se desprende del Global Entertainment and Media Outlook 2013-2017, elaborado por PwC que incluye las perspectivas de crecimiento de las principales industrias que conforman el sector de entretenimiento y medios -publicidad online, televisión, prensa, revistas, mercado editorial, cine, videojuegos, etc.- en España y en el mundo hasta 2017. Por subsectores, el de la publicidad en buscadores es el que acumula la mayor cuota de mercado y alcanzó en 2012 los 639 millones de dólares. Le sigue la publicidad display con 385 millones de inversión en 2012 (Puromarketing.com, 2013).

Las relaciones entre las empresas publicitarias y los anunciantes deben ser buenas, si se quiere recuperar y dinamizar el sector publicitario. Por ello hasta 11 asociaciones que representan a los principales actores en el sector de la publicidad en España, entre anunciantes, agencias de marketing, agencias de medios y medios de comunicación han firmado el primer Código de Conducta Comercial para mejorar las relaciones entre las compañías del sector a través de una mejora de la "transparencia" entre ellas y como código de buena conducta. El Código hace hincapié en la coordinación entre las compañías que intervienen en la comunicación comercial en una situación en el que las relaciones entre ellas estaban "gastadas", según Fernando Ocaña, representante de la Asociación Internacional de la Publicidad (IAA por sus siglas en inglés), organismo que ha coordinado la elaboración del código. A su juicio, con la aprobación del código "no gana ni pierde nadie, sino que gana el sector en general", y demuestra de esta forma su "madurez" (Elconfidencial.com, 2007).

Son diversas las acciones desarrolladas con el objetivo de internacionalizar la publicidad española, muestra de ello es el proyecto “Gazpacho Connection”, un HUB digital con las mejores estrategias y piezas de comunicación concebidas en España, y una plataforma e-CRM para compartirlas de forma selectiva con anunciantes, consultores, periodistas y profesores 
del resto del mundo. Esta iniciativa se enmarca en el Plan Sectorial de la Publicidad con el que ICEX apoya a este sector en España y que a su vez responde al objetivo estratégico del organismo público de promover la exportación de servicios. Las asociaciones de las que parte la iniciativa son: Asociación Española de Agencias de Comunicación Publicitaria (AEACP), Asociación de Agencias Digitales (AD), Interactive Advertising Bureau (IAB), Club de Creativos (CdC), Asociación de Agencias de España (ADE) y Asociación de Productoras de Cine Publicitario (APCP). "Gazpacho Connection" tiene su antecedente en la celebración de COME 09, un encuentro con más de 100 anunciantes norteamericanos que se celebró en la sede neoyorkina del Instituto Cervantes y que permitió a las principales agencias, mostrar y demostrar sus capacidades (Icex.es, 2013).

Conviene recordar el importante efecto económico que la publicidad tiene en la economía de los países y el escaso eco que este valor generado tiene en los medios y de las razones de estos hechos. La industria de la publicidad es, por tanto, una herramienta empresarial, nada más, ni nada menos. El efecto indiscutible que las ideas creativas han supuesto en el valor que la publicidad aporta al anunciante ha hecho a veces olvidar el objetivo real de la inversión en publicidad: la mayor rentabilidad del cliente. Se ha antepuesto el medio (la creatividad) al fin (los negocios de las empresas anunciantes); si la creatividad no fuera útil, no se necesitaría publicidad (Riesgo, 2005:125).

\subsubsection{El sector publicitario en Castilla y León}

Según el estudio La Publicidad en Castilla y León: Informe Profesional del sector 2011, en primer lugar, debemos destacar el pronunciado desequilibrio existente entre las distintas regiones de España. En la cúpula de la inversión publicitaria se posicionan Madrid y Cataluña, que concentran elevados volúmenes de inversión de los anunciantes y lideran conjuntamente el mercado de la publicidad en España. La media de inversión por cada anunciante madrileño asciende a los 542.426 euros y a 198.549 por cada anunciante catalán. La diferencia con otras comunidades como Galicia, Extremadura y Castilla y León, que se sitúan a la cola en el listado de regiones según su inversión por anunciante, es más que notoria (Fernández Blanco, 2011:18).

Castilla y León, en concreto, cuenta con un elevado número de anunciantes, pero también con la menor inversión media por anunciante de toda España, únicamente 15.233 euros en 2010. Estos indicadores evidencian que el mercado castellanoleonés está conformado por un elevado número de anunciantes, pero con bajos presupuestos publicitarios. Las excepciones más significativas a nivel de inversión son La Junta de Castilla y León, Grupo Pascual o Cadbury.

Durante el año 2010, la inversión de las empresas con sede social en Castilla y León ascendió hasta casi 217 millones de euros (incremento del $+11,21 \%$ con respecto a 2009 , datos del i2P), frente al $+1,7 \%$ de crecimiento experimentado por el sector a nivel nacional. Además, entre 2008 y 2010 el crecimiento acumulado en el sector castellano-leonés fue de un 35,5\%, algo que contrasta con el brusco descenso de la inversión publicitaria en España. El estudio muestra, por tanto, que este sector regional 
no funciona al mismo ritmo que a nivel nacional que "los datos desvelan que el mercado castellano-leonés no está sujeto a los vaivenes publicitarios y sus desequilibrios, ya que no se rige por las tendencias macropublicitarias” (Elmundo.es, 2011).

El sector está marcado por una gran red de pequeñas agencias que carecen de un posicionamiento diferenciado en el mercado, en tanto que ofertan un servicio total, un "todo para todos". A la falta de posicionamiento se suman otros problemas claramente identificados por sus protagonistas: fuga de grandes anunciantes de la región, falta de cultura de comunicación de los anunciantes, ausencia de asociacionismo y corporativismo, intrusismo profesional, competencia desleal de los medios, entre los más importantes. Sin embargo, estos puntos de fricción conviven con otras características propias de la región, como son la cercanía, el conocimiento profundo del mercado y los consumidores y la adaptación a sus clientes.

Atendiendo a los distintos sectores, el de la alimentación fue el que más invirtió (por incluirse en el mismo el Grupo Pascual en el estudio i2P), con 118,4 millones de euros. Le sigue, aunque con notable diferencia, el de las bebidas, con 19,2 millones de euros. Ambos sectores son muy significativos en la región, que presenta uno de sus puntos económicos fuertes en los mismos. Alimentación está liderado por marcas de referencia como Pascual, Vive Soy, Trident, Bifrutas, Hall, Facundo, Helios o Tierra de Sabor. Por su parte bebidas está encabezado por Bezoya, Altos de Tamarón, Yllera, Viña Pedrosa o dehesa la Granja.

En tercer lugar, se posiciona el sector de servicios públicos y privados (16,9 millones de euros de inversión), que incluye todo tipo de campañas de organismos públicos desde ayuntamientos, diputaciones y la propia Junta de Castilla y León, así como todo tipo de asociaciones y servicios privados, que constituyen un importante músculo de la actividad publicitaria de la región.

Gráfico 1. Distribución de la inversión publicitaria por sectores en Castilla y León en 2010 (en miles).

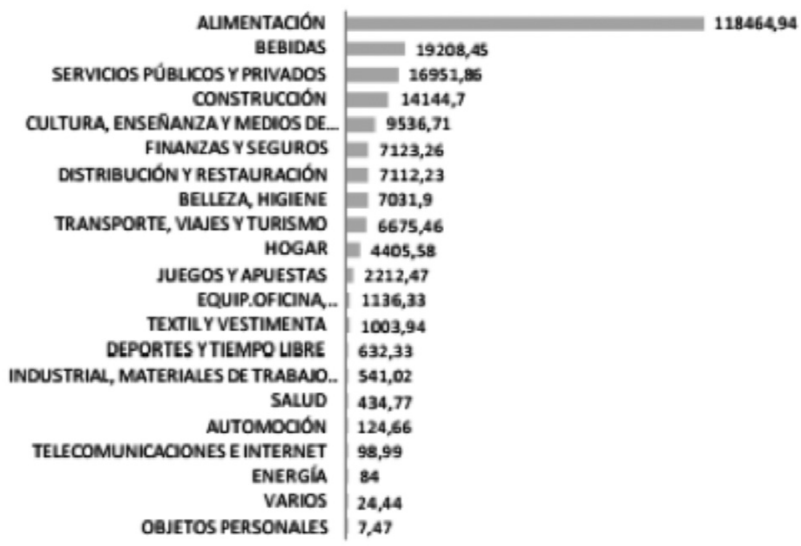

Fuente: Estudio La Publicidad en Castilla y León. Datos i2P 
Como ya se apuntó con anterioridad, Grupo Pascual, Cadbury y la Junta de Castilla y León son los mayores anunciantes de la región, los que se desmarcan de esa ingente cantidad de anunciantes de la comunidad que Grupo Pascual se posiciona como primer anunciante de Castilla y León con 89.431.280 euros de inversión en 2010, un 18.25\% más que en 2009. Cadbury, por su parte, ha incrementado su presupuesto el 166\%, llegando a un total de 31.110.290 euros, lo que le sitúa como el segundo anunciante más importante en la región. Sin embargo, la Junta de Castilla y León ha reducido su inversión de 19.866.870 euros en 2009 a 15.543.890 euros en 2010 (un 20,97\% menos de inversión).

Gráfico 2. Los 50 mayores anunciantes por inversión en Castilla y León en 2010.

\begin{tabular}{|c|c|c|c|}
\hline Anunciantes & Año $2010(000$ 's) & Añ̄o 2009 (000\%s) & $\mathbf{X}$ inc. 10/09 \\
\hline GRUPO LECHE PASCUAL & 89431.28 & 75629.42 & 18.25 \\
\hline CADBURY & 31130.29 & 11677.65 & 166.58 \\
\hline JUNTA CASTLLA LEON & 15543.83 & 19668.87 & -20.97 \\
\hline ALTAMIRA CONSTRUCCIONES & 8310.37 & 11264.27 & -26.22 \\
\hline FACUNDO BLANCO & 4118.3 & 6320.91 & -34.85 \\
\hline FORTE CONSULTING & 3706.38 & 2004.66 & 84.89 \\
\hline MARCOS SALAMEANCA (IAMONONE) & 3381.91 & 2687.4 & 25.84 \\
\hline GRUPOTEJEDOR & 2836.48 & 2967.51 & 4.42 \\
\hline AYTO.VALLADOUD & 2811.5 & 2919.6 & .3 .70 \\
\hline CALA ESPANA & 2642.52 & 1732.57 & 52.52 \\
\hline POSTQUAM COSMETIC & 2453.89 & 3174 & -22.69 \\
\hline GENERAL BETS ESPANA & 2206.43 & & \\
\hline VEKA & 2092.39 & 0.48 & 435814.58 \\
\hline CALA DUERO & 2052.66 & 1171.02 & 75.29 \\
\hline LABORATORIOS LAROME & 1633.85 & & \\
\hline PAGOS DEL REY & 1546.69 & 183031 & -15.50 \\
\hline HELIOS & 1414.25 & 3963.65 & -64.32 \\
\hline GRUPO EL ARBOL DISTRIBUCION Y SUPERMERCADOS & 1251.56 & 566.86 & 120.79 \\
\hline MARCO ANTONIO MARTIN TORQUEMADA & 1144.44 & & \\
\hline GRUPO PHARMADUS & 1058.63 & 22.4 & 4626.03 \\
\hline S.ATLOS CURROS & 1036.49 & 71.87 & 1342.17 \\
\hline CYSDA & 1027.57 & & \\
\hline INSTITUCOON EDUCATIVA SEK & 966.52 & 1087.35 & -11.11 \\
\hline STRATO AG & 950.6 & 1367.69 & -30.50 \\
\hline SILOE ARTE Y BIBLIOFILLA & 929.56 & 893.22 & 4.07 \\
\hline SORIA NATURAL & 925.31 & 1704.24 & -45.71 \\
\hline LEGUMBRES LUENGO & 915.08 & 667.33 & 37.13 \\
\hline TELEVISION CASTILLALEON & 831.88 & 695.82 & 19.55 \\
\hline FABRICACION MODERNA DEL HOGAR & 797.82 & 261.31 & 205.32 \\
\hline MAYA CORREDURLA SEG. & 704.63 & 1.96 & 35850.51 \\
\hline SCAVOUNI & 693.06 & 520.15 & 33.24 \\
\hline CALA BURGOS & 643.03 & 562.98 & 14.22 \\
\hline BODEGAS HNOS.PEREZ PASCUAS & 618.44 & 667.06 & .7 .29 \\
\hline NOVADIET & 566.14 & & \\
\hline ALDEADUERO & 554.52 & & \\
\hline PAREOES & 532.95 & 197.32 & 170.09 \\
\hline FED.ESPANOLLA DONANTES SANGRE & 510.62 & 395.43 & 29.13 \\
\hline DECOLUX & 508.9 & 5457.22 & -90.67 \\
\hline GALLETAS GULLON & 496.05 & 81897 & -39.43 \\
\hline BODEGA Y UNEDOS FERNANDEZ RIVERA & 487.23 & 438.83 & 11.03 \\
\hline BODEGA MUSEUM & 394.54 & 245.26 & 60.87 \\
\hline FUNERARIA SAN MIGUEL & 356.94 & 33652 & 6.07 \\
\hline GALA SANITARIOS & 324.83 & 227.43 & 42.83 \\
\hline GORMAZ INMOQILARIA & 315.7 & 556.49 & -43.27 \\
\hline AGRICOLA CASTELLANA & 308.78 & 142.99 & 115.95 \\
\hline BODEGAS FARINA & 301.85 & 81.31 & 271.23 \\
\hline AYTO.LEON & 284.06 & 393.99 & -27.90 \\
\hline DIPUTACION VALLADOUID & 284.04 & 336.95 & -15.70 \\
\hline DIPUTACION SALAMANCA & 278.1 & 302.07 & .7 .94 \\
\hline EL ADELANTO & 261.47 & 47.82 & 446.78 \\
\hline
\end{tabular}

Fuente: Estudio La Publicidad en Castilla y León. Datos i2P 
En cuanto a la inversión de los anunciantes de Castilla y León por medios de comunicación, el estudio i2P señala que el medio en el que más presupuesto invierten es la televisión, con un 47,29\% del total. Esto se corresponde con la gran inversión que efectúan en este medio las grandes marcas anteriormente mencionadas (Grupo Pascual, Cadbury y Junta de Castilla y León), ya que la mayor parte de los anunciantes acude a la prensa para difundir sus campañas. Como el presupuesto de estos anunciantes es significativamente menor que el de los tres grandes, la prensa se sitúa en el segundo lugar con un $20,32 \%$ de la inversión total. En tercer lugar, se posiciona la radio, con un 17,36\%, seguida por Internet, con un $7,19 \%$, revistas con un 3,39\% y dominicales con un 2,62\%. En las últimas posiciones encontramos el medio exterior con un $1,23 \%$ de la inversión total y al cine, con únicamente un 0,06\%.

Gráfico 3. Inversión de los anunciantes de Castilla y León por medios en 2010.

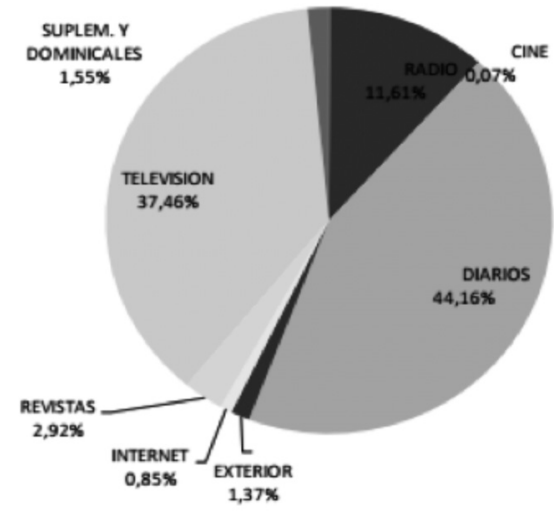

Fuente: Estudio La Publicidad en Castilla y León. Datos Infoadex

Por lo tanto, a través de ambos estudios podemos hacernos una idea de la situación del sector publicitario en Castilla y León y concluir que la tendencia nacional no arrastra al sector publicitario regional. Aunque cada uno de los estudios considere una muestra diferente, se constata que la inversión publicitaria en Castilla y León aumentó (en mayor o menor medida según la fuente) en 2010 y que la prensa, la televisión y la radio son los medios convencionales que mayor cuota reciben por parte de los anunciantes, frente al cine, por ejemplo, donde la inversión es mínima.

\subsubsection{El sector publicitario en Valladolid}

Los Premios de Publicidad de Castilla y León han estado liderados por las agencias de Valladolid entre los años 2007 y 2011. Un total de 10 agencias vallisoletanas han conseguido 22 Premios Oro en estos últimos cinco años. Tras Valladolid, los publicistas de Salamanca, con 9 Oros y los burgaleses, con 4 . Y en la cola las provincias de Ávila, Segovia y Soria, que no cuentan con ningún galardón (TribunaAvila.com, 2011). 
Centrándonos en Valladolid, cabe destacar las agencias "Metáfora Comunicación”, "RQR Comunicación” y “Trama Comunicación y Diseño", las cuales han recibido, cada una, 4 de estos máximos galardones. Les siguen "4C Comunicación”, "Bi Comunicación”, “Estudio Pobrelavaca” y “Salvi design”, con 3 Premios Oro cada una. Estos premios se dividen en distintas categorías, en cada una de las cuales se adjudica un Premio Oro y un Premio Plata, en concreto: diseño de identidad corporativa; publicidad gráfica en prensa y revistas; packaging; redes sociales y web 2.0; radio; diseño aplicado en artes gráficas; publicidad exterior; eventos y acciones especiales; spots y audiovisuales y web y soportes interactivos. Además, existen otras cuatro categorías premiadas únicamente con el Oro: Premio Honorífico; anunciante solidario; mejor campaña Castellano y Leonesa y mejor campaña estatal, además de una “Categoría Young” para jóvenes creativos.

Tras conocer el amplio abanico de categorías y disciplinas publicitarias que incluyen estos Premios y comprobar que el jurado está compuesto por distintos expertos y grandes conocedores del sector publicitario, podemos afirmar que el liderazgo de las agencias vallisoletanas en cuanto al número de galardones puede considerarse una muestra bastante evidente de su gran capacidad. Por lo tanto, concluimos que, a nivel de Castilla y León, Valladolid es la provincia que más ha demostrado la valía de su sector publicitario en términos cualitativos.

\section{Objetivos}

Este trabajo se centra en el análisis del sector publicitario (compuesto en su mayoría por pymes) en Castilla y León y concretamente en Valladolid. Se define el sector de la comunicación y la publicidad, la tipología de empresas que ofrecen estos servicios y, en última instancia, la actitud y el comportamiento de las pymes hacia los mismos.

En este artículo profundizamos en los tipos de agencias de publicidad existentes en nuestro entorno y en cómo es la actividad de trabajo de dichos profesionales de la comunicación, teniendo en cuenta que en la mayoría de casos funciona el binomio pyme-agencia, y como muestra la investigación un porcentaje alto de las empresas de publicidad analizadas son pymes.

\section{Hipótesis}

Las hipótesis de partida son las siguientes:

1. La madurez del mercado obliga al sector publicitario vallisoletano y, por ende, a las agencias que lo conforman, a organizarse a partir de una estructura departamental que permita ofrecer una gran variedad de servicios, ya que la mayor parte de las agencias de publicidad de Valladolid son de "servicios plenos".

2. Las nuevas tecnologías y el auge de Internet han provocado que tanto la oferta como la demanda de acciones de comunicación por parte de las Agencias de Publicidad y de las pymes respectivamente sean, hoy en día, las más representativas de la actividad publicitaria. 
3. Las pymes cada vez son más conscientes de la importancia de la comunicación corporativa para sus negocios, por lo que cada vez más acuden a profesionales de la publicidad y la comunicación en busca de soluciones, se dejan asesorar y mantienen con estos proveedores una relación fluida y cordial.

\subsection{Metodología de investigación}

Para conocer con mayor detalle la situación del sector publicitario en Valladolid, procedemos a describir la metodología y resultados de la investigación llevada a cabo gracias a la colaboración de las agencias de publicidad/comunicación de la ciudad.

Para realizar esta investigación, tras barajar distintas posibilidades como la entrevista personal, se optó por la modalidad cuantitativa, a través de la herramienta de la encuesta.

Se utilizaron encuestas con "cuestionario autoadministrado", "en el que la figura del entrevistador no existe, se envía por correo o por e-mail" (Pedret et al. 2000:196) y es el encuestado quien accede al cuestionario y lo cumplimenta sin asistencia. En este caso, el envío de las mismas se hizo a través de correo electrónico. Este tipo de encuestas, según Grande y Abascal (2001:202), se caracteriza por los siguientes aspectos: debe enviarse una carta que explique lo que se espera del encuestado y la finalidad de la investigación; debe explicarse que el cuestionario es anónimo; deben proporcionarse instrucciones claras; deben utilizarse preguntas cerradas; las preguntas más convenientes son las dicotómicas; y el diseño y la presentación deben ser atractivas, pues así aumenta la tasa de respuesta.

Además debemos señalar que utilizamos los servicios de la página web www.encuestafacil.com. Gracias a esta plataforma, pudimos diseñar nuestro cuestionario combinando preguntas de todo tipo: abiertas, con respuesta desplegable, con posibilidad de elección de varias opciones..., en todo momento siguiendo un orden e incluyendo, incluso, líneas o párrafos explicativos durante el desarrollo de la misma.

\subsubsection{Universo, muestra y método de muestreo}

El universo, finalmente, y tras recabar datos de distintas fuentes (Páginas Amarillas, QDQ, Foroingenio, eInforma, Camerdata, Confederación Vallisoletana de Empresarios y Asociación Vallisoletana de Agencias de Publicidad), hemos considerado que está conformado por un total de 38 agencias. Durante la realización de esta búsqueda, una de las conclusiones que obtuvimos es que es imprescindible contrastar este tipo de informaciones, ya que, en muchos casos, nos encontramos con empresas que habían desaparecido o que, en otras ocasiones, habían cambiado de nombre. Por otro lado, ningún directorio está absolutamente completo; el compendio de todas las fuentes ha sido elemental a la hora de conocer todos los miembros del universo. 
Tras el envío de las encuestas a los 38 miembros del universo inicial, se obtuvieron 27 encuestas contestadas. De las 27, tres fueron duplicados y una de ellas fue enviada en blanco, por lo que, finalmente, la muestra consta de 23 encuestas. Es decir, se ha obtenido una tasa de respuesta del 60,52\%. Dentro de las 23 encuestas contestadas y aceptadas para el estudio, hay que señalar que dos de ellas están incompletas. Uno de los encuestados informó a través de correo electrónico de un error a la hora de realizar la encuesta y de que buscaría el momento de volver a contestarla tan pronto como le fuera posible. Esto no ocurrió, pero hemos considerado esta encuesta válida, ya que un tercio de las preguntas fueron correctamente contestadas. En el caso de la otra encuesta incompleta, el encuestado no explicó por qué se detuvo y no finalizó la misma, pero un tercio de preguntas contestadas nos sirven también para hacernos una idea de la propia agencia, ya que ese tercio de respuestas se corresponden, precisamente, con el primer bloque de preguntas: "Bloque I: Sobre la Agencia/Consultoría de Comunicación/Publicidad”. Por lo tanto, de las 23 encuestas recibidas, podemos concluir que un 91,3\% están completas y, por lo tanto, un $8,7 \%$ no lo están.

Gráfico 4. Distribución de las encuestas en base a la respuesta obtenida.

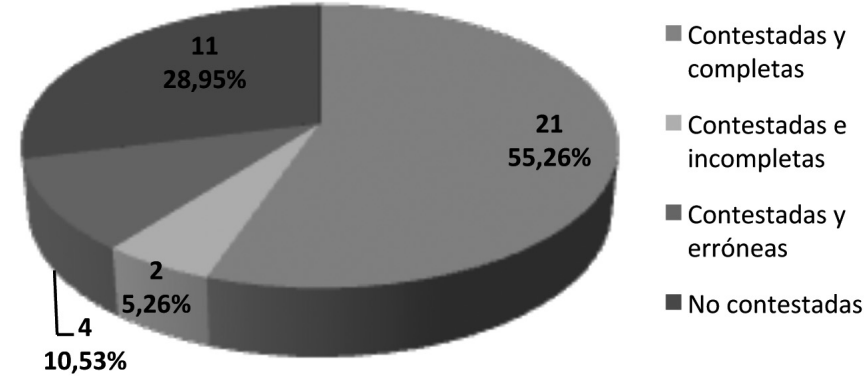

Fuente: Elaboración propia

\section{Resultados}

Una vez descrita la metodología y explicados los detalles de la investigación, procedemos a explicar los resultados obtenidos.

En cuanto al bloque I de preguntas "Sobre las agencias de publicidad de Valladolid" éstos fueron los resultados más interesantes:

Sobre la antigüedad de las agencias de publicidad de Valladolid, puede decirse que las agencias de Valladolid son, en su mayoría, de reciente creación o, lo que es lo mismo, que el sector publicitario vallisoletano es relativamente “joven". Más de la mitad de las agencias de la ciudad, un $61 \%$, fueron creadas hace menos de 10 años. En concreto, un $39 \%$ tiene 5 años o menos de trayectoria y un $22 \%$ nació entre 6 y 10 años atrás. Otro $22 \%$ de las agencias vallisoletanas tiene una antigüedad 
de entre 11 y 15 años, frente al 13\%, cuya actividad lleva desarrollándose entre 16 y 20. Por último, las más escasas son las agencias “decanas", las impulsoras de la actividad publicitaria en Castilla y León y con mayor antigüedad (más de 20 años), que sólo representan un $4 \%$ de la muestra. Esta "juventud” de las agencias de publicidad de Valladolid tiene relación con la reciente creación de nuevas agencias especializadas en el ámbito online.

Respecto a la tipología de las agencias de publicidad de Valladolid, señalamos que muchos de los encuestados no quisieron clasificar su agencia dentro de una única modalidad, si no que citaron de forma conjunta varias de las opciones adjuntas en la pregunta. Esto constata la diversificación en la oferta de estas empresas, donde la clasificación que viene haciéndose tradicionalmente ya no se ajusta de forma óptima, sino que las propias agencias se reinventan y crean nuevas formas de negocio. La tipología predominante de agencias de publicidad en Valladolid es la de "agencias de servicios plenos o servicios generales" (un 65\% de las agencias dijo identificarse en esta denominación), seguida por "estudios de diseño” y “agencias especializadas en nuevas tecnologías”, con un 35\% de citaciones respectivamente. Por último, las actividades menos predominantes son las de "agencias especializadas en otras actividades relacionadas con la publicidad: marketing promocional, buzoneo...” (17\% de menciones), al igual que "agencia de comunicación” (17\% de menciones).

Cabe señalar que el 52,18\% de los encuestados citó varias modalidades de agencia a la hora de definir su negocio. El restante $47,82 \%$ declaró que su agencia podía enmarcarse perfectamente dentro de cualquiera de las tipologías aportadas. $34,78 \%$ del total de los encuestados afirmó trabajar en una agencia de servicios plenos, un 8,69\% en una "especializada en nuevas tecnologías" y un 4,33\% dijeron ser, exclusivamente, un "estudio de diseño".

Gráfico 5. Tipología de agencias de publicidad en Valladolid.

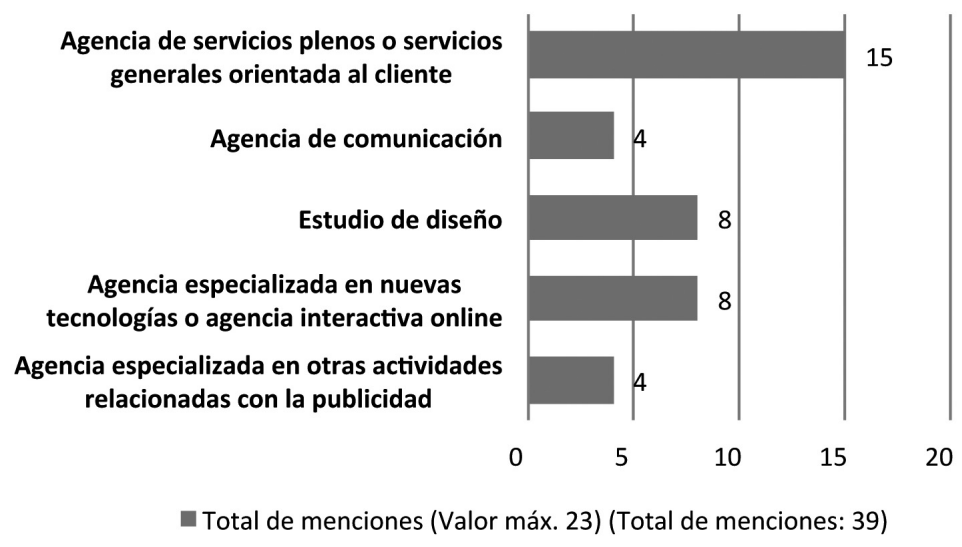

Fuente: Elaboración propia 
En cuanto a los servicios ofrecidos por las agencias, los resultados encajan con los servicios que prestan las agencias de Valladolid según este estudio. Los más ofrecidos son el "Diseño publicitario" (mencionado por el 87\% de los encuestados, es decir, 20 de las 23 agencias lo ofrecen), "Campañas integrales" (19 menciones, 83\% de los encuestados) y "Publicidad" (74\% de la muestra). En cuanto a servicios de producción, un 70\% de encuestados afirmó proveer a sus clientes de "servicios de producción audiovisual” y un 65\% citó la producción gráfica. El 70\% de los encuestados declaró participar en la “organización de eventos, ferias y convenciones", el 57\% citó los servicios de "Publicidad exterior” y el 52\% de "Marketing online". Los últimos puestos son para los servicios relacionados con la "evaluación de campañas y acciones" (5 menciones, 22\%) y los "estudios de mercado" (3 menciones, 13\%), seguidos, finalmente, por tres servicios adicionales que algunas agencias dijeron prestar (1 mención por cada uno de los servicios): "Naming + branding", "Regalo publicitario" e "Instalaciones museísticas, espectáculos audiovisuales, accesibilidad para personas con discapacidad sensorial...”.

Gráfico 6. Principales servicios ofertados por las agencias de Valladolid.

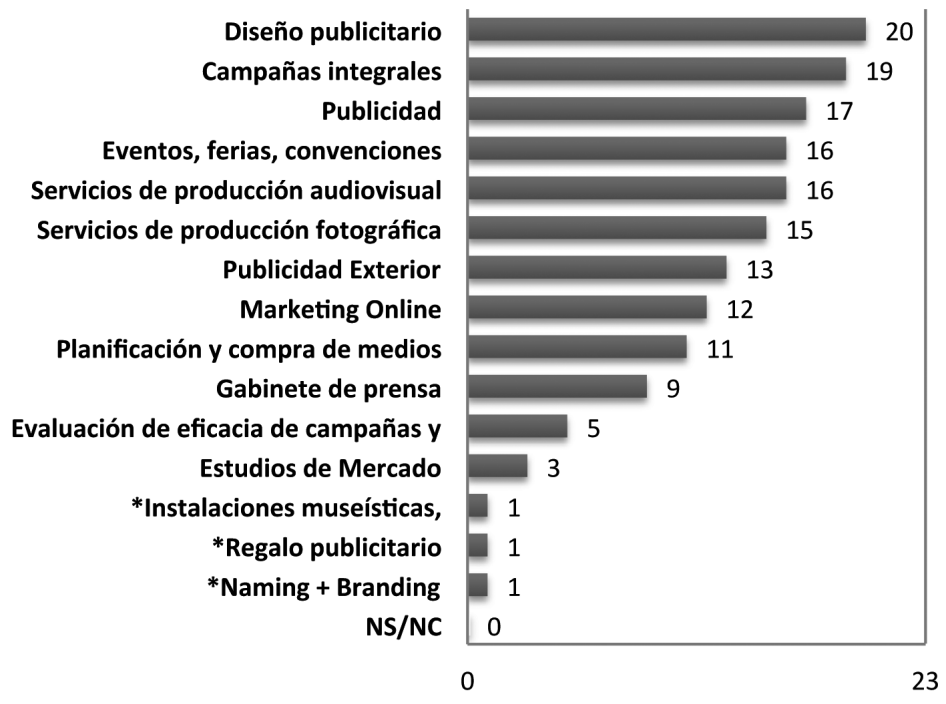

- Total de menciones (Valor máx. 23) (Total de menciones: 159)

Fuente: Elaboración propia

En cuanto al posicionamiento, indicamos que el posicionamiento buscado por las agencias tampoco se ajusta a un único parámetro, tal y como demuestran los resultados de este estudio, en el que todas las agencias señalaron varias de las posibilidades como descriptoras de su posicionamiento. Este posicionamiento va relacionado con la tipología de agencia en la que dicen identificarse, ya que el posicionamiento estratégico más mencionado es el de "servicios plenos orientados al 
cliente" (el 65\% lo mencionaron) junto con "calidad" (65\%). La "experiencia” y la "atención al cliente, cercanía y relación familiar" (39\% respectivamente) son otros de los valores más importantes para las agencias a la hora de posicionarse, seguidos por la especialización en diseño (35\%) y por ser una “empresa familiar” o estar "especializada en otras áreas: eventos, marketing promocional..." (26\% respectivamente). Llama la atención que, a pesar del auge de Internet, únicamente un 17\% de la agencias menciona que estar especializada en "Marketing Online" es un gran valor de su posicionamiento estratégico. La "especialización en Pymes” está a la cola con un 9\% junto a varias categorías a las que únicamente mencionó un $4 \%$ de la muestra respectivamente (es decir, una agencia mencionó cada categoría), las cuales son las relacionadas con el posicionamiento en base al ámbito geográfico ("vínculo directo con el ámbito local y regional" o "posicionamiento geográfico amplio"). Con un $4 \%$ de menciones encontramos también "Ayudamos a las Pymes a que vendan más", "Radio", “Confianza, confidencialidad y servicio" y “Diseño web”, citadas por los propios encuestados.

Gráfico 7. Posicionamiento estratégico de las agencias de Valladolid.

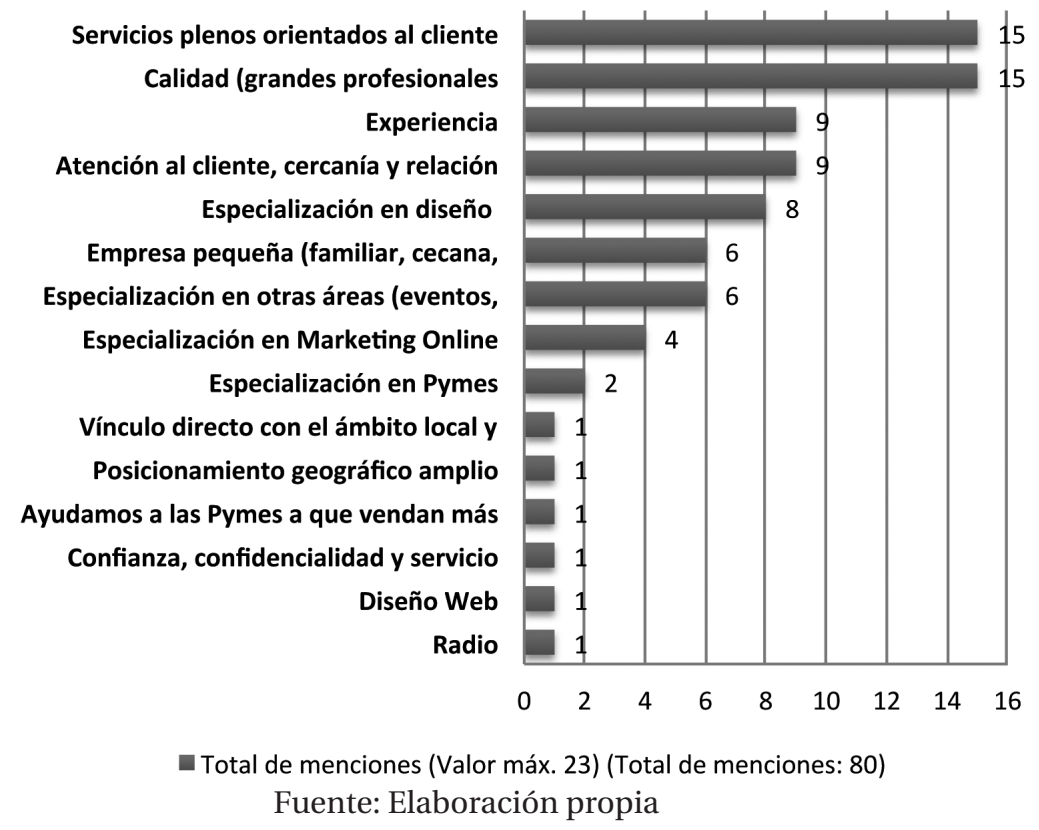

En lo relativo a las características empresariales de las agencias, en cuanto a la forma jurídica de las agencias de Valladolid, podemos concluir que la predominante en las agencias de Valladolid es la de Sociedad Limitada (70\%), seguida por la de Empresario Individual (17\%), Comunidad de Bienes (9\%) y Sociedad Laboral Limitada (4\%). La tipología de Sociedad Anónima no ha sido citada por ninguno de los miembros de la muestra. 
Respecto a la plantilla y facturación de las agencias de publicidad, aproximadamente la mitad de la muestra, un $47,82 \%$ consta de entre 1 y 5 personas, un 26,08\% de entre 6 y 10 y un 8,69\% consta de entre 11 y 15 asalariados. Únicamente un $4,76 \%$ tiene más de 15 asalariados en su plantilla y otro 4,34\% señaló no poder aportar una cifra concreta ya que "depende de la temporada". La mayor parte de las plantillas se componen de 3 personas (30,43\%).

Gráfico 8. Número de personas que conforman las plantillas de las agencias de publicidad de Valladolid.

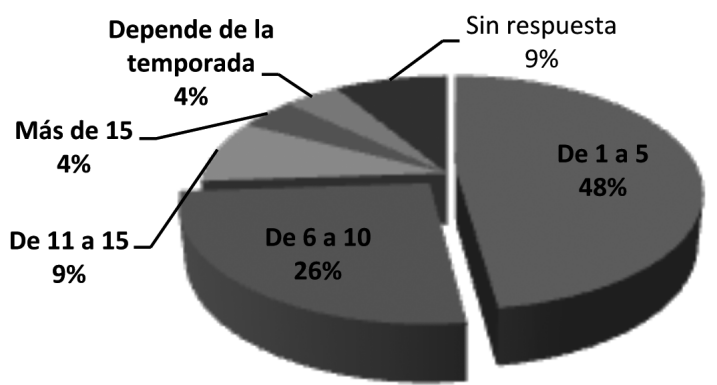

Fuente: Elaboración propia

Por lo tanto, podemos concluir que las agencias de Valladolid, en su mayoría (73,9\%), son microempresas (menos de 10 empleados y volumen de facturación menor de 2 millones de euros, según la Recomendación 2003/361/CE de la Comisión Europea). Para completar esta afirmación, analizaremos el volumen de facturación de las agencias de Valladolid. La gran mayoría facturan por debajo de los 2 millones de euros (65,21\%), el 17,39\% factura entre 1 y 3 millones de euros y un 8,69\% respondieron NS/NC o no contestaron a la pregunta, respectivamente. Por lo tanto, la mayor parte de las agencias de publicidad de Valladolid son microempresas.

Gráfico 9. Nivel medio de facturación de las agencias de Valladolid en 2012.

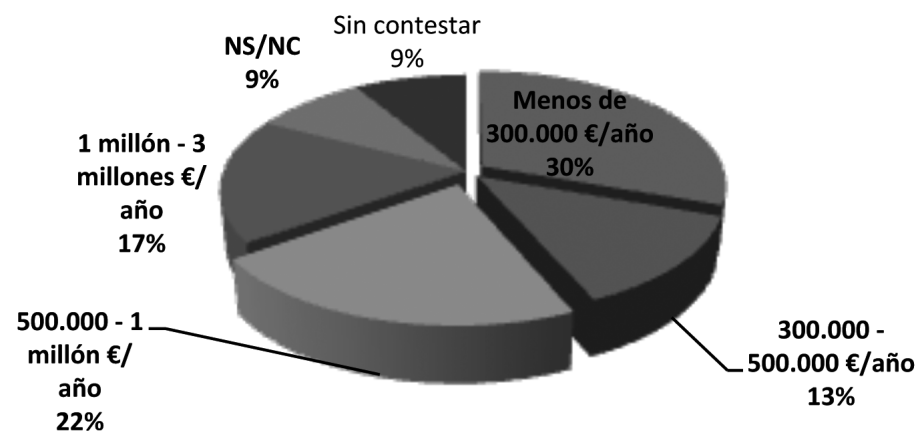

Fuente: Elaboración propia 
Continuamos con la información acerca de los empleados de la agencia, en concreto, su edad. Podemos afirmar que el sector económico de la publicidad es joven en Valladolid, ya que la media de edad de profesionales de esta actividad es de 34 años. El 52,17\% de ellos tiene entre 31 y 40 años, seguidos por un 34,78\% que tiene 30 años o menos. Por lo tanto, un 86,95\% de los profesionales de la publicidad y la comunicación de Valladolid tienen 40 años o menos. Únicamente un 4,35\% de los trabajadores tiene más de 41 años. En el 71\% de las empresas los empleados son técnicos y casi en la misma proporción, en un $67 \%$, poseen formación universitaria. Un $0 \%$ de las agencias declararon que en su plantilla hubiera algún empleado sin formación de alguno de estos dos tipos.

Por lo tanto, podemos afirmar que los profesionales de la publicidad y la comunicación de Valladolid disponen de formación en su totalidad. Además, la formación universitaria se corresponde con la función después desempeñada, ya que el 52,17\% de las agencias dijeron tener en su plantilla Licenciados en Publicidad y Relaciones Públicas. La segunda titulación más presente en las agencias vallisoletanas es una muy vinculada con el diseño y la creatividad, Bellas Artes (34,78\%), seguida por titulaciones relacionadas con la gestión empresarial, como son Económicas o Empresariales (30,43\%) y Administración y Dirección de Empresas (21,74\%). Licenciados en derecho (17,39\%), Informáticos (17,39\%) y Periodistas $(13,04 \%)$ son los siguientes profesionales más presentes en las agencias de publicidad de Valladolid. Resulta curioso que los Licenciados en Comunicación Audiovisual estén presentes únicamente en el 4,34\% de las agencias, debido a la relación entre sus estudios y las labores, por ejemplo, de producción. Esto se justifica con el alto índice (73\%) de subcontratación de este servicio en concreto por parte de las agencias de publicidad de Valladolid. Otras titulaciones señaladas por los encuestados son Ciencias del Trabajo, Ingenierías, Psicología y Medicina (únicamente una agencia por titulación, es decir, un 4,34\% de las agencias mencionó cada una de estas titulaciones, respectivamente). 
Gráfico 10. Titulaciones universitarias de los empleados de las agencias de Valladolid.

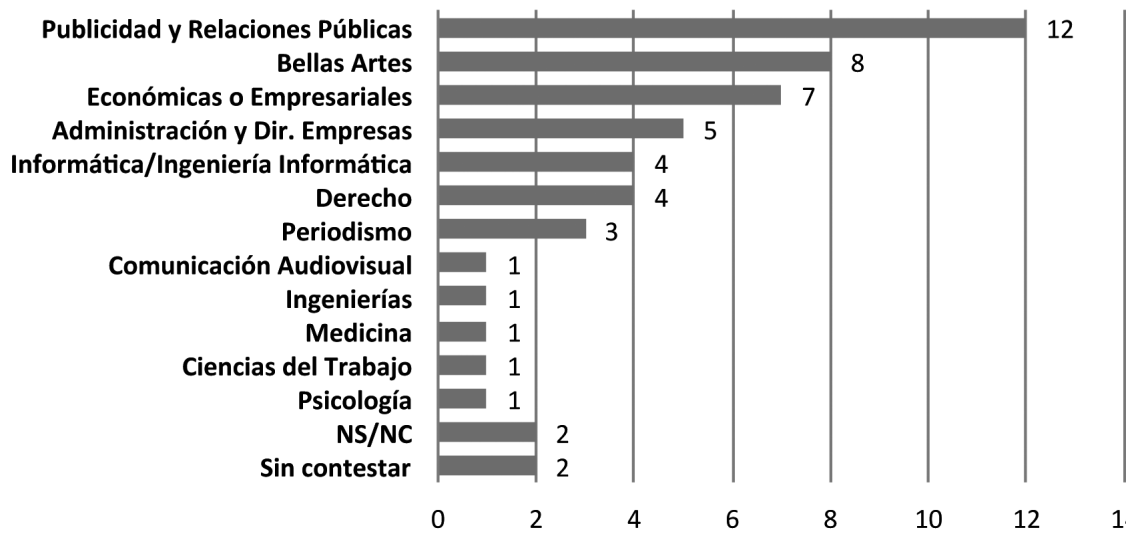

número de agencias que disponen de empleados con esta titulación. (Valor máximo: 21) (Número de menciones: 50)

Fuente: Elaboración propia

Gráfico 11. Evolución de la formación de los empleados de las agencias de publicidad de Valladolid.

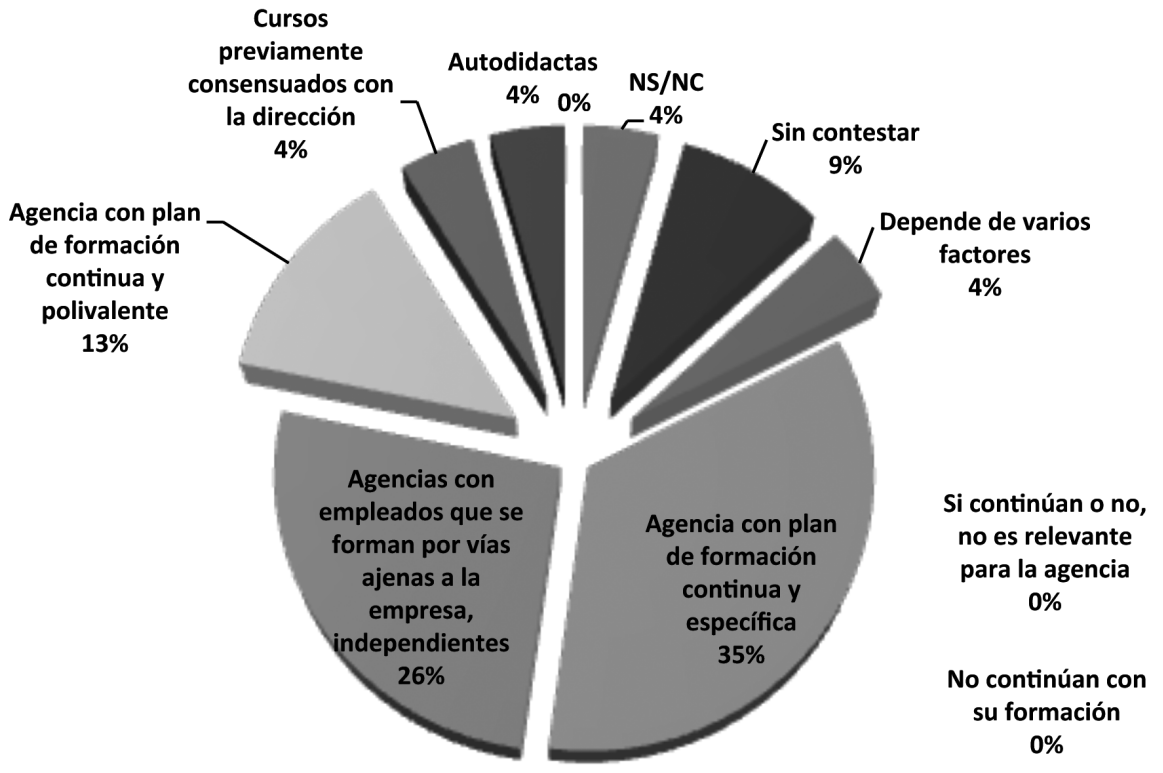

Fuente: Elaboración propia 
Hemos descrito los servicios que ofrecen las agencias de Valladolid, pero un 96\% de las mismas afirmaron subcontratar algún servicio, frente a un $4 \%$ que dijo no hacerlo.

En relación a esos servicios subcontratados (outsourcing), la producción audiovisual fue la más citada (73\% de los encuestados dijeron subcontratarla), junto con la producción gráfica (45\%). Les siguen los estudios de mercado (27\%), la publicidad exterior (23\%) y la evaluación de campañas y acciones (18\%). Un 14\% de las agencias declaró subcontratar servicios de diseño publicitario (folletos, imagen corporativa...) y de marketing online (otro 14\%). El 9\% de las agencias subcontrata servicios para la organización de eventos, ferias o convenciones y un $5 \%$ de las agencias que subcontratan servicios citaron: servicios de publicidad, planificación y compra de medios, gabinete de prensa y la opción de NS/NC. El único servicio que ninguna agencia declaró subcontratar fue el de la organización de campañas integrales, algo que parece bastante lógico. Sobre el motivo por el que subcontratan estos servicios, las agencias señalaron, en su mayoría, que se debe simplemente a que la agencia no dispone de ese servicio (45\%), además de para "lograr mejores resultados" (36\%). "La agencia no está especializada o debidamente cualificada para realizar esta actividad" (27\%), "saturación en momentos concretos" (18\%) o coste menor (9\%) fueron otras de las respuestas aportadas. Por último, un 5\% de las agencias seleccionaron la opción NS/NC, así como la de "Los servicios de producción los realizan empresas especializadas, imprentas, productoras, etc.”, señalada por uno de los encuestados.

Gráfico 12. Servicios subcontratados por las agencias de publicidad de Valladolid.

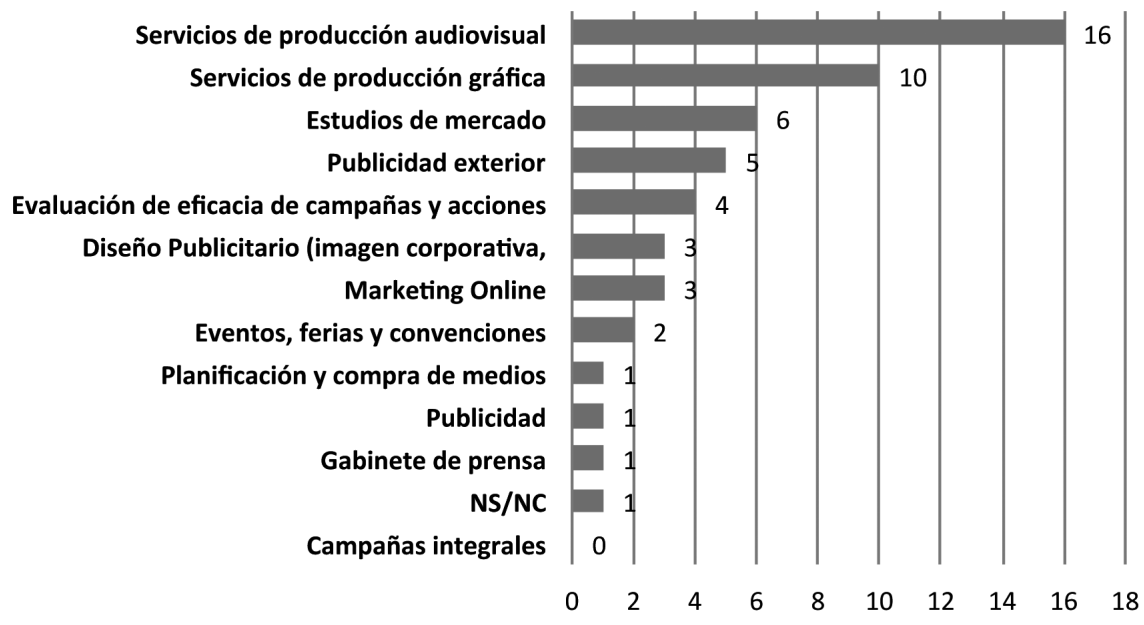

- Total de menciones de servicios subcontratados (Valor máx. 22) (Total de menciones: 53)

Fuente: Elaboración propia 
En cuanto a la organización interna de las agencias de publicidad, en Valladolid, predominan notablemente las agencias divididas por departamentos o áreas (52,17\%) frente a las que se organizan en función de los clientes o cuentas $(4,34 \%)$. Existen, por otro lado, agencias que utilizan ambos métodos para estructurarse $(21,74 \%)$, es decir, un sistema combinando cuentas/departamentos. Por último, un 8,69\% de las agencias no cuentan con una estructura determinada.

La división de la estructura interna de las agencias más común es "por departamentos o áreas", describimos cuáles son esos departamentos existentes en las agencias de publicidad de Valladolid. Entre las agencias que están organizadas según áreas o departamentos, los más comunes son el de Administración (existente en el 84,21\% de las agencias divididas en departamentos) y el de Creatividad (presente en el 78,94\% de las mismas). En el 47,36\% de las agencias se distingue un área dedicada al Marketing Online y en un 42,1\% existe un departamento destinado a Eventos y Producción. La Planificación de medios es la siguiente área que más predomina, ya que está presente en el 36,34\% de la muestra, seguido por Cuentas (31,57\%). Los menos frecuentes son el de Relaciones Públicas (15,73\%) y los de Investigación y Planificación estratégica, presentes únicamente en una de las agencias encuestadas respectivamente, lo que se traduce en un 5,26\% de presencia para cada uno, al igual que el área de Sistemas, señalado adicionalmente por uno de los participantes en el estudio. El área de marketing directo o promocional es el único que ninguna de las agencias encuestadas indica distinguirse en su estructura $(0 \%)$.

Respecto a la valoración del sector publicitario, en una escala de 0 a 10, donde entendemos 0 como "situación pésima” y 10 como "situación óptima", el 26,03\% de los encuestados optó por un "aprobado raspado" de la situación eligiendo como valoración un 5. En torno a este "suficiente" obtenemos las puntuaciones positivas y negativas, las cuales, estas últimas, consiguen un total del 43,41\% de la valoración de las agencias. Es decir, casi la mitad de los encuestados consideran la situación del sector de la publicidad negativo. Un 8,69\% la valoró con un 2, un 21,74\% con un 3 y un 13,04\% de la muestra con un 4. En el otro polo encontramos a un 17,39\% de los encuestados, que dan una valoración positiva a la situación del sector. Un 8,69\% la puntuó con un 6 y un 4,34\% con un 7 y un 8 respectivamente. Las puntuaciones más bajas y más altas, los extremos de los polos, no fueron escogidas por ningún encuestado y uno de los participantes en el estudio eligió la opción NS/NC.

La valoración de la situación actual es, en general, más negativa que positiva. Pero, ¿̨cuál es la previsión para el sector publicitario para los próximos años? No muy alentadora. Según las agencias de publicidad de Valladolid, la situación empeorará significativamente, o eso afirma el $21,73 \%$ de ellas, junto a un 17,39\% que opina que empeorará ligeramente. Es decir, el 39,13\% de las agencias vallisoletanas hacen un pronóstico negativo de la situación de su actividad en los próximos años. En el punto medio, encontramos a un 17,39\% de encuestados que opina que el sector se mantendrá estable. El 21,73\% de las agencias considera que el sector mejorará en los próximos años, un 17,39\% considera que sensiblemente y un 4,34\% que bastante. El 13,04\% de los encuestados prefirió no posicionarse ni hacer ningún pronóstico. Por lo tanto, la perspectiva de futuro para el sector publicitario, según los profesionales de esta actividad en Valladolid, no es demasiado positiva. 
Gráfico 13. Perspectiva de futuro del sector de la publicidad según los profesionales de las agencias de publicidad de Valladolid en 2012.

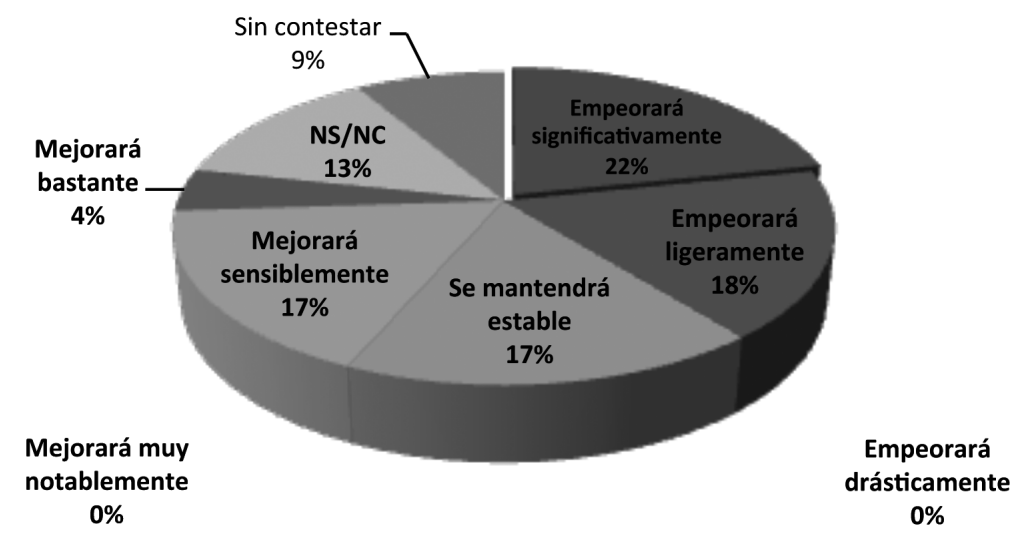

Fuente: Elaboración propia

A continuación, presentamos los resultados extraídos del bloque II de preguntas: "Sobre los clientes de las agencias (pymes)":

En primer lugar, tras la investigación podemos afirmar que la media de clientes (en general, no solamente pymes) con los que trabajaron durante el año 2011 las agencias de publicidad en Valladolid es de 57,05 clientes. Consideramos este dato no significativo, ya que las diferencias de datos aportadas por las distintas agencias son bastante notorias, por lo que las agruparemos en rangos para tener una imagen más real de la realidad de las carteras de clientes de las agencias vallisoletanas.

La mayor parte de las agencias de publicidad de Valladolid trabajó, en el último año, con entre 1 y 40 clientes. En total, un $56,52 \%$ de ellas. Por lo tanto, vemos que, aunque la media alcanza los 57,05 clientes, no es la tendencia dominante. Un 17,39\% de las agencias sí se encuentran en el rango que comprende el valor de la media. En concreto, cuentan en su cartera de clientes con entre 41 y 80 de ellos. Únicamente un 4,34\% tuvo entre 81 y 120 clientes y un 8,69\%, o lo que es lo mismo, dos de las agencias, alcanzaron cifras de más de 120 clientes. En concreto, señalaron haber trabajado con 200 y 350 clientes. Estas cifras son las que hacen que el valor de la media se eleve y no sea representativo de la realidad del sector publicitario de Valladolid. Cabe señalar que un $4,34 \%$ de las agencias prefirieron no aportar este dato y señalaron que era "confidencial".

Por otro lado, la forma de ganar clientes más citada, mencionada por un 78,25\% de las agencias, fue gracias a su experiencia y trabajos anteriores. Esta experiencia y trabajos previos parecen satisfacer a sus clientes, ya que el segundo motivo 
que alegan a la hora de conseguir nuevas cuentas es gracias a otros clientes, que les recomiendan (citado por un 69,55\% de las agencias). El factor personal es importante, ya que además de la recomendación de los clientes, los contactos personales que tiene la propia agencia son el tercer motivo que el 47,82\% de la muestra menciona. Mediante la promoción y distintas acciones comerciales (34,78\%) y el hecho de ser ya muy conocidos en el panorama de la publicidad y la comunicación (13,04\%) son los siguientes motivos. Por último, un 4,34\% respectivamente cita las "acciones especiales" y el "posicionamiento SEO en buscadores" como formas de lograr nuevos clientes.

En cuanto a los sectores a los que pertenecen las pymes con las que trabajan las agencias de Valladolid, la mayor parte de ellas dicen trabajar con pymes pertenecientes al sector de "hostelería y restauración" (60,87\%), "alimentación" (56,52\%), "administraciones públicas" (52,17\%), "enseñanza, cultura y medios de comunicación” (47,83\%) y "servicios privados" (47,83\%). A estos sectores les siguen el de "Salud" (39,13\%), “ONG, Fundaciones, Asociaciones" (34,78\%), "Industria" (34,78\%), “Construcción” (34,87\%) y “Belleza y Cosmética” (34,78\%). Los sectores menos citados por las agencias son el de "Moda y textil" y "Energía", con un 26,08\% de menciones respectivamente, el de "Internet y telecomunicaciones", con un $21,74 \%$ y, con diferencia, el de Finanzas (banca, seguros...), con únicamente un 4,34\% de menciones. Independientemente del motivo (puede que simplemente no acudan a agencias de publicidad o que, debido a su gran volumen de negocio o su carácter de gran empresa o multinacional, como en el caso de ciertas marcas de moda o empresas de banca, seguros o telefonía, requieran los servicios de agencias ubicadas en ciudades como Madrid), podemos concluir que los sectores que menos contratan los servicios de las agencias de Valladolid son el de "Internet y telecomunicaciones" y el de "Finanzas". En el lado opuesto, los sectores que más requieren los servicios de las agencias de publicidad de Valladolid son el de la "Hostelería”, la "Alimentación”, las "Administraciones públicas”, la "Enseñanza, cultura y medios de comunicación” y los "Servicios privados".

Los motivos por los que una pyme acude a una agencia de comunicación (un 65,21\% de menciones) son: la venta de productos, llevar a cabo acciones promocionales puntuales y la gestión de su imagen corporativa. Esto muestra que, además de la búsqueda del beneficio económico de forma directa, las pymes son conscientes de que la imagen corporativa es un valor importante que también se traduce en beneficios, aunque sea a más largo plazo. El cambio de posicionamiento ( $26,08 \%$ de las agencias lo mencionan), la incursión en nuevos mercados (21,74\%), la ampliación de la cartera de clientes $(13,04 \%)$ y la mejora de la presencia en Internet (SEO, Redes Sociales, Web...) $(4,34 \%$ de menciones) son otros de los motivos citados por los que las Pymes acuden las agencias de Valladolid. Un 4,34\% no supo definir los objetivos de estas pymes cuando acuden a solicitar sus servicios.

En lo referente a los servicios más demandados por las pymes, las agencias de publicidad señalan el "Diseño y producción web” (mencionado por el 65,21\% de la muestra), "Diseño de catálogos, folletos, papelería...” (60,86\%), "Diseño gráfico" (60,86\%), “Campañas publicitarias” (52,17\%) e "Identidad corporativa” (47,83\%). Estos servicios más demandados se co- 
rresponden, claramente, con los servicios que las agencias proveen y que ya analizamos con anterioridad, cuando concluimos que el servicio más generalizado era el de "Diseño" (el 87\% de las agencias de Valladolid lo ofrecen), las "Campañas integrales" (83\%) y la "Publicidad" (ofrecido por un 74\%). Por lo tanto, podemos decir que oferta y demanda se ajustan en el sector de la publicidad en Valladolid. Las agencias saben lo que sus clientes demandan más y se ajustan a esos requerimientos. Los servicios menos solicitados, según los resultados de esta investigación, son los de "producción fotográfica y audiovisual" y "gabinete de prensa" (citado por un 8,69\% de la muestra únicamente) y, sobre todo, la "decoración y ambientación de espacios", que sólo un 4,34\% de la muestra señaló como servicio solicitado por las pymes.

Gráfico 14. Servicios más demandados por las Pymes a las agencias de publicidad de Valladolid.

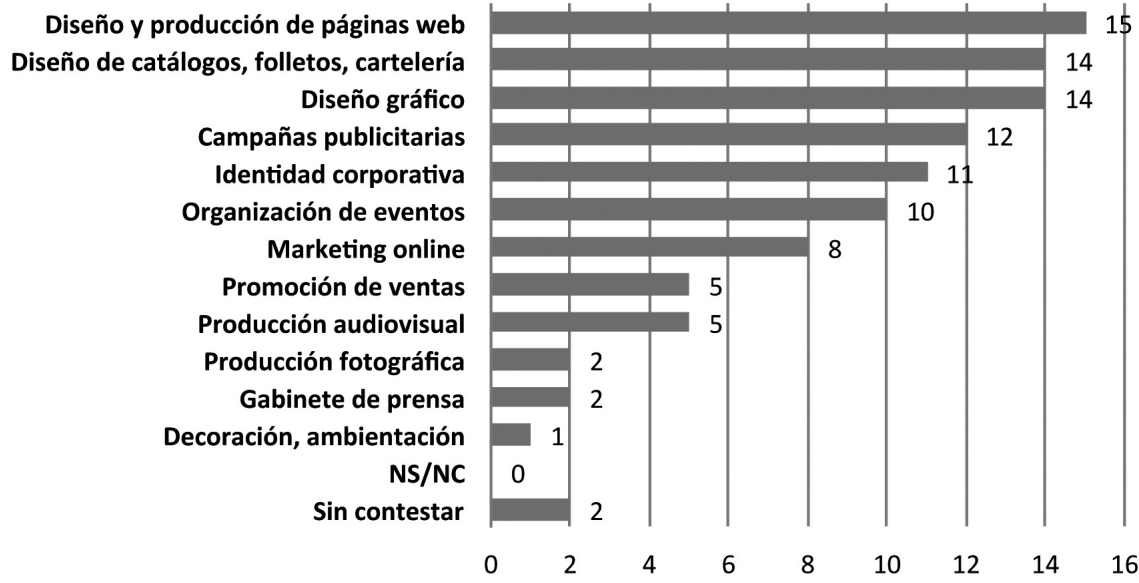

- Agencias que citan cada uno de los servicios como "demandado por las Pymes" (Valor máx. 21) (Total de menciones: 87)

\section{Fuente: Elaboración propia}

En cuanto a los medios de difusión más solicitados y preferidos por las pymes, en primer lugar está Internet (60,87\% de las agencias lo consideran como uno de los más solicitados), seguida por diarios y suplementos y la radio, con un $43,47 \%$ de agencias que los mencionan respectivamente. A mayor distancia, con una diferencia significativa de número de agencias que los consideran soportes de verdadera referencia a la hora de analizar la contratación de las pymes, encontramos el medio exterior (17,39\%), las revistas (13,04\%), las acciones Below The Line (13,04\%) o la televisión (8,69\%). Por último, el cine y, citada por uno de los encuestados, la imprenta, sólo son señalados por un $4,34 \%$ de la muestra respectivamente. Internet es, como ya hemos señalado anteriormente, el medio más solicitado por las pymes. Por ello, vamos a concretar qué tipo de acciones son las más solicitadas en este medio ya que, además, el 100\% de las agencias que contestaron a este 
grupo de preguntas del estudio trabajan con este medio. Lo más solicitado es la creación del sitio web de la pyme (mencionado por el $69,56 \%$ de las agencias) y, en segundo lugar, además de su creación, la posterior gestión de la misma (52,17\%). El posicionamiento en buscadores (43,48\%), las inserciones publicitarias en distintos espacios $(34,78 \%)$, el desarrollo de una campaña concreta $(34,78 \%)$ y la creación y distribución de virales $(26,08 \%)$ son las siguientes acciones más requeridas por las pymes según las agencias de publicidad. La creación de un blog y la gestión de sus contenidos fue citada por el 17,39\% de las agencias como uno de los servicios más requeridos por sus pymes clientes y, finalmente, con un 4,34\% de citaciones respectivamente, encontramos la "presencia y gestión de redes sociales" (relacionado con la labor de Community Manager, tan de moda en la actualidad) y las "campañas de CPC". Por lo tanto, todo lo relacionado con la creación y gestión de páginas web es lo más solicitado por las pymes, aunque la estrategia del control del posicionamiento en buscadores está ganando terreno a acciones más "tradicionales" como la simple inserción de piezas publicitarias en distintos espacios de la red.

Gráfico 15. Acciones más solicitadas por las Pymes dentro del medio Internet según las agencias de Valladolid (2012).

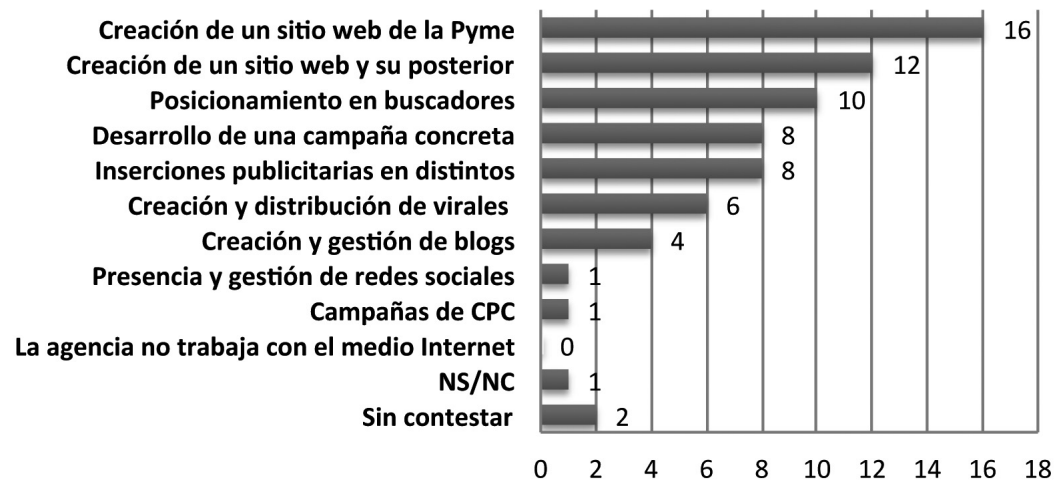

- Agencias que consideran cada servicio como uno de los más solicitados por las Pymes (Valor máx. 21) (Total menciones: 67)

Fuente: Elaboración propia

Al preguntar por el presupuesto medio que las pymes invierten en acciones de publicidad y comunicación a cada una de las agencias, el 30,43\% de ellas prefirió no aportar una cifra concreta. La amplitud de la pregunta y la gran variedad de pymes hace que esta respuesta sea comprensible. Sin embargo, en el resto de casos, obtuvimos distintas respuestas, cuya media es de 4.435,71€ al año. Pero las respuestas son bastante dispares, existe una concentración de respuestas entre los 1.000 y los 4.000 euros (el 26,09\% de la muestra), pero nos encontramos con valores extremos y tan lejanos como $300 €$ y $12.000 €$. Todo depende de qué agencia aporte el dato y de qué pyme se tome como referencia. Si dividimos las respues- 
tas por rangos, comprobamos que la mayor parte de respuestas, el 46,15\% de aquellos que se han aventurado a dar una cifra, posicionan esta inversión anual por debajo de los $2.000 € /$ año. El resto de posibilidades no superan el 10\%, por lo que podemos decir que la mayor parte de las pymes invierten 2.000 euros o por debajo de esta cantidad en comunicación y/o publicidad al año.

La relación entre las agencias y sus clientes, en este caso en concreto, las pymes, puede desarrollarse de distintas formas. Nosotros hemos querido distinguir entre dos modalidades, como son la de partner (la agencia actúa como un asesor y socio. Tiene capacidad de maniobra) y proveedor (la agencia simplemente satisface las necesidades y solicitudes del cliente). Aunque la opción NS/NC también estaba disponible, ninguno de los encuestados la ha seleccionado, sino que el $52,18 \%$ de la muestra dijo que la relación entre su agencia y sus clientes era de “partner", mientras que un 39,13\% aseguró predominar una relación "proveedor”. Apuntamos que un 8,69\% de la muestra no contestó a la pregunta.

A la hora de analizar la relación entre la agencia y las pymes, además del rol y consiguiente capacidad de maniobra de las agencias, hemos procurado recopilar la valoración que éstas atribuyen a la misma. En una escala de 1 a 5, donde 1 es "muy mala", 3 “cordial” y 5 "muy buena”, la opción mayoritariamente escogida por la muestra fue la 4, "buena”, con un 43,48\%. En segundo lugar, la opción "muy buena”, que fue señalada por el 39,13\%. Por último, un 8,69\% escogió la opción 3 "cordial” y otro 8,69\% no contestó a esta pregunta. Por lo tanto, es evidente que la relación entre pymes y agencias es muy positiva, distendida y más que cordial. Además, predomina la capacidad de maniobra de las agencias, algo muy positivo a la hora de buscar verdaderas soluciones para las empresas, en lugar de limitarse a "obedecer" a lo que éstas ya han decidido.

Gráfico 16. Valoración de la relación Pyme-Agencia por parte de las agencias de Valladolid (2012).

- Número de agencias que atribuyen cada valoración a su relación con las Pymes (Valor máx. 21)

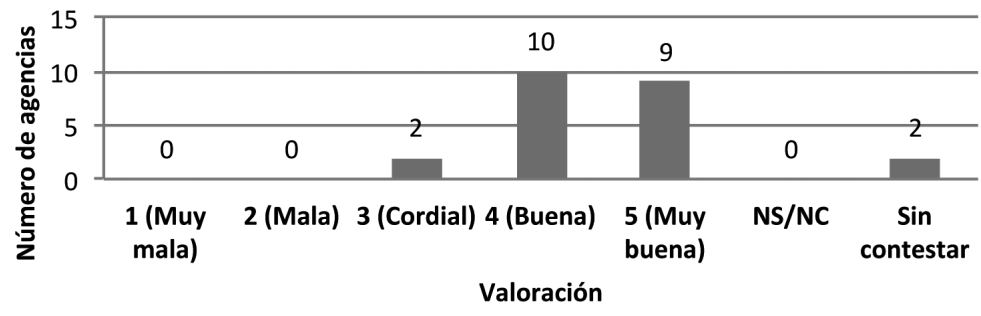

Fuente: Elaboración propia

Para que esta facilidad de aceptación de ideas y consejos sea real, es importante que las agencias sean capaces de arriesgar, de buscar formas creativas de comunicarse y no ajustarse únicamente a las estrategias tradicionales. En cuanto a esta 
cuestión, el 39,13\% de las agencias de publicidad de Valladolid consideran que prima la búsqueda de seguridad y que, por este motivo, las pymes se ajustan a las acciones convencionales. Únicamente un 13,04\% de la muestra declara que la tónica predominante es la búsqueda de creatividad y el desarrollo de acciones innovadoras y, por ende, más arriesgadas. El $39,13 \%$ restante opina que ambas posturas se dan en la misma proporción. Por lo tanto, podemos destacar que el hecho de que en raras ocasiones nos encontremos con iniciativas realmente sorprendentes en la ciudad de Valladolid se debe a esa poca predisposición de los clientes a asumir riesgos y diferenciarse de los demás a través de acciones creativas.

Gráfico 17. Valoración de las agencias de publicidad de la facilidad de aceptación de ideas y consejos por parte de las Pymes (2012).

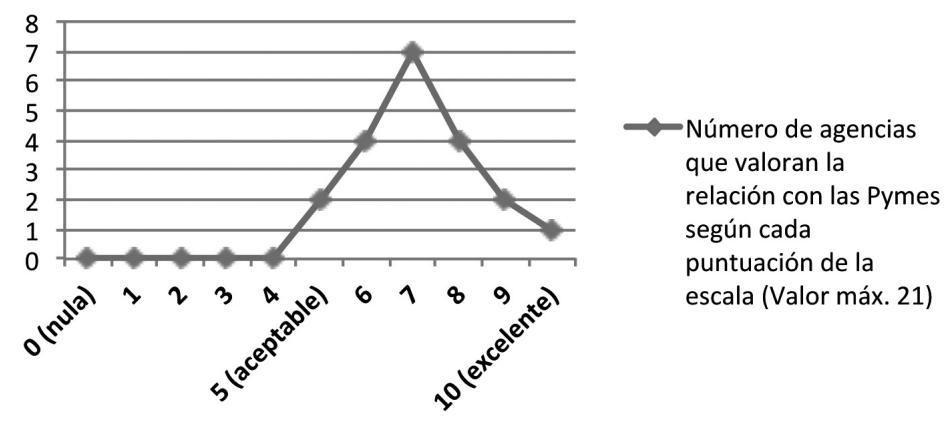

Fuente: Elaboración propia

\section{A modo de conclusión}

La crisis económica que afecta a los mercados, evidentemente, incide sobre el sector de la publicidad. La inversión en publicidad a nivel nacional ha disminuido e, incluso, el tradicional "medio estrella", la televisión, ha sufrido los recortes presupuestarios de los anunciantes.

El sector publicitario castellano-leonés no se ve afectado por el vaivén del español y, además, los anunciantes de la región apuestan cada vez más por esta actividad y creen en la necesidad de invertir en ella para darse a conocer. Por ello, no es que simplemente la inversión no haya disminuido como ocurre a nivel nacional, sino que en Castilla y León la inversión en publicidad ha aumentado. No obstante, la inversión en Castilla y León es, con gran diferencia, inferior a otras comunidades autónomas, como Cataluña o Madrid. Castilla y León consta de una ingente cantidad de anunciantes, pero la inversión de los mismos, salvo de los tres grandes (Grupo Pascual, Cadbury y la Junta de Castilla y León) es muy baja. Esta situación confirma una de las hipótesis planteada en el estudio: Las pymes cada vez son más conscientes de la importancia de la comunicación corporativa para sus negocios, por lo que cada vez más acuden a profesionales de la publicidad y la comunicación en busca de soluciones, se dejan asesorar y mantienen con estos proveedores una relación fluida y cordial. 
El medio en el que más invierten los anunciantes castellano-leoneses es la televisión según el estudio i2P (que considera al Grupo Pascual dentro de la región) y los diarios según Infoadex (que lo considera fuera de Castilla y León). Aunque la televisión sea el medio que más dinero recibe, la realidad es que la prensa es al que más anunciantes regionales acuden para difundir sus acciones. En cuanto a sectores, en Castilla y León el más significativo es el de la alimentación, debido a la pertenencia al mismo de los grandes grupos señalados anteriormente. Le sigue el de las bebidas y el sector de servicios públicos y privados, donde se encuentra el otro gran anunciante, la Junta de Castilla y León (aunque ha reducido su inversión en el último año).

En Valladolid, ciudad referente en el sector publicitario regional, observamos, en primer lugar, la dificultad de encontrar información pertinente cuanto más estrechamos el cerco geográfico. En primer lugar, las agencias de publicidad de la ciudad son de reciente creación en su mayoría, pero que también existen, todavía, algunas de las pioneras del sector en la región. Además, los profesionales que trabajan en estas pymes son jóvenes, tienen formación específica y continúan con ella mientras desempeñan su trabajo. La mayor parte de las agencias ofrecen a sus clientes casi todos los servicios relacionados con la publicidad y la comunicación, entre los que destacan, de forma específica, los relacionados con el diseño y la publicidad propiamente dicha. Esta capacidad de ofrecer servicios plenos es uno de los pilares del posicionamiento estratégico de las agencias, además de valores como la calidad o la experiencia. Esta conclusión se relaciona con el cumplimiento de otra hipótesis mencionada en el estudio: La madurez del mercado obliga al sector publicitario vallisoletano y, por ende, a las agencias que lo conforman, a organizarse a partir de una estructura departamental que permita ofrecer una gran variedad de servicios, ya que la mayor parte de las agencias de publicidad de Valladolid son de "servicios plenos".

Aunque por no disponer de todos los servicios requeridos por los anunciantes o, simplemente, para lograr mejores resultados, la mayor parte de las agencias de publicidad de Valladolid subcontratan todo lo relativo a la producción y, también, los estudios de mercado.

Por otro lado, la mayor parte de estas empresas realizan acciones a nivel nacional y muchas de ellas trabajan con todos los medios pero, sin duda, el más citado por las mismas es Internet. Esto coincide con el hecho de que, además, las acciones en Internet sean las más solicitadas por las pymes en la actualidad. Este planteamiento corresponde a la confirmación de otra hipótesis formulada: las nuevas tecnologías y el auge de Internet han provocado que tanto la oferta como la demanda de acciones de comunicación por parte de las agencias de publicidad y de las pymes respectivamente sean, hoy en día, las más representativas de la actividad publicitaria.

\section{Referencias bibliográficas}

Aedemo (2010): “Radiografía de la industria publicitaria en España”. Disponible en: http:/ /www.aedemo.es/aedemo/images/stories/area_comunicacion/radiografia_ind_\%20publicitaria\%20_espana.pdf [Consultado el 20/12/2013]. 
Asociación Española de Agencias de Comunicación Publicitaria (2011): "Situación de la planificación estratégica en España”. Disponible en: http://www.agenciasaeacp.es/publicaciones/docs/SPE_Espana3.pdf [Consultado el 28/04/2012].

Blanco, J. M (2011): “La inversión en publicidad crece un 11\% y el sector alimentario acapara la mitad”, Elmundo.es, 29 de noviembre de 2011. Disponible en: http://www.empresasdepublicidad.com/noticias/la-inversion-en-publicidad-creceun-11-y-el-sector-alimentario-acapara-la-mitad/ [Consultado el 20/04/2012].

Europa Press (2007): "El sector de la publicidad en España firma un convenio de buenas prácticas comerciales para mejorar la transparencia”, Elconfidencial.com, 17 de noviembre de 2007. Disponible en: http://www.elconfidencial.com/ cache/2007/11/12/60_sector_publicidad_espana_firma_convenio_buenas_practicas_comerciales.html [Consultado el 28/12/2013].

Europa Press (2013): "El mercado español de la publicidad online crecerá un 4,9\% en los próximos cinco años", Puromarketing.com, 29 de octubre de 2013- Disponible en: http://www.puromarketing.com/14/18441/mercado-espanolpublicidad-online-crecera-proximos.html [Consultado el 29/12/2013].

Fernández Blanco, E. et al. (2011): La Publicidad en Castilla y León. Informe profesional del sector. Salamanca: Foro Ingenio y Universidad Pontifica de Salamanca.

Grande, I. y Abascal, E. (2001): Fundamentos y técnicas de investigación comercial. Madrid: Esic.

Informe Infoadex 2013. Disponible en: http://www.infoadex.es/InfoAdex_Resumen_Est_Inv_2013.pdf [Consultado el 15/01/2014].

Informe Sector de la publicidad (2007). Disponible en: http://www.clustergrafico.com/files/aie/8_sector_publicidad.pdf. [Consultado el 20/12/2013]

Ley General de Publicidad 34/1988 (artículo 10). Disponible en: http://www.boe.es/boe/dias/1988/11/15/pdfs/A3246432467.pdf. [Consultado el 16/01/2014].

Ministerio de Educación de España (2012): “¿Quién es y qué hace la agencia?”. Disponible en: http://recursos.cnice. mec.es/media/publicidad/bloque4/pagl.html. [Consultado el 18/01/2012].

Pedret, R. et al. (2000): La investigación comercial como soporte del marketing. Bilbao: Universidad Deusto.

Riesgo, A. (2005): "La economía de la publicidad. Generación de valor de la actividad publicitaria a la economía”, Telos Cuadernos de Comunicación e Innovación, n.64, pp.125-138. Disponible en: http://sociedadinformacion.fundacion.telefonica.com/telos/cuadernograbar.asp@idarticulo=2\&rev=64.htm [Consultado el 28/12/2013].

\section{Webs consultadas}

Asociación Vallisoletana de Agencias de Publicidad: http://fecosva.com/asociaciones/avep/avep.htm [Consultado el 05/02/2012]. 
Cámara de Comercio de Valladolid: www.camaravalladolid.com/ [Consultado el 22/03/2012].

Confederación Vallisoletana de Empresarios: www.cve.es [Consultado el 22/03/2012].

Foro Ingenio: www.foroingenio.com/asociados.html. [Consultado el 19/01/2012].

Icex.es: http://www.icex.es/icex/cda/controller/pageICEX/0,6558,5518394_5593093_6366453_4643227_0_-1_p5712643,00. html [Consultado el 29/12/2013]. 